\title{
CERCAL SENSORY DEVELOPMENT FOLLOWING LASER MICROLESIONS OF EMBRYONIC APICAL CELLS IN ACHETA DOMESTICUS ${ }^{1}$
}

\author{
JOHN S. EDWARDS,* SU-WAN CHEN,* AND MICHAEL W. BERNSł \\ * Department of Zoology, University of Washington, Seattle, Washington 98195 and $\ddagger$ Department of Developmental and Cell Biology, \\ University of California, Irvine, California 92717
}

\begin{abstract}
The hypothesis that pioneer fibers, which develop relatively early in the differentiation of insect appendages, serve to organize the peripheral sensory nerves was tested by ablating apical regions of the cercal rudiments in embryos of Acheta domesticus. Multiple nerve bundles rather than the normal middorsal and midventral pair of nerves were formed within the cercus following ablation of the cercal tip before pioneer fiber differentiation, but the cercal nerve was normal when lesions were made after formation of the pioneer fiber tracts and associated glia. These results indicate a necessary morphogenetic role for the pioneer fibers.
\end{abstract}

A guidance function has been proposed for sets of neuron-like cells, the so-called pioneer fibers, that appear in insect appendages during early embryonic development in advance of sensory neuron differentiation (Bate, 1976; Edwards, 1977; Keshishian, 1980). The basis for this inference is the relative timing of their differentiation, the pioneer fibers appearing before the elongation of appendage primordia and forming central projections when the distances separating periphery and center are smallest. It is proposed that, by following these pioneer fibers, later differentiating sensory axons reach their central targets along pathways that, in the course of organogenesis, become considerably longer and more tortuous than that required of the pioneers, but it remains to be shown that pioneer fibers are necessary for the organization of the definitive sensory nerve. Proof of their necessity requires demonstration that the sensory axons arising from functional sensilla late in embryogenesis do not become organized into a normal nerve in the absence of the pioneer fiber pathway.

In this study, we have used the abdominal cerci of the house cricket, Acheta domesticus. These posteriorly placed sensory appendages contain purely sensory nerves formed by aggregation of different axons that arise from

\footnotetext{
${ }^{1}$ Dedicated in memory of Professor William T. Keeton of Cornell University who was an inspiration as both a teacher and researcher in neurobiology.

This work was supported by National Institutes of Health Grants NB 07778 (J. S. E.) and GM 23445 (M. W. B.). Irradiation was conducted in the Laser Microbeam Facility under Biotechnology Resource Grant RRO 1192-1. We thank Drs. J. Palka and M. Meyer for their critiques of the manuscript.
}

cell bodies situated in the epidermis, as is typical of insects. Since the cerci lack intrinsic muscles, they provide appropriate material for the study of guidance of sensory nerves alone. The normal embryonic development of the cercal sensory system has been described elsewhere (Edwards and Chen, 1979).

The initial attempts to produce localized lesions in embryonic cercal rudiments were made with tungsten microneedles. Eggs were penetrated under saline and the tip of the cercal rudiment was pricked so that apical cells or the entire structure was ablated. In most embryos so treated, the damaged embryonic epithelium became attached to the egg membranes at the site of puncture and further development was grossly distorted as a result of the adhesion. The few embryos that completed development after puncture and hatched with reduced or no cerci had also suffered damage to the anal and adjacent areas during the egg penetration and were not able to develop beyond the midpoint of the first instar. Since mechanical damage could not be localized to the cercal tip, an alternative approach using an ultraviolet laser microbeam was used for the work described below.

We report here that ablation of the apical region of embryonic abdominal cerci, at a time before the centripetal processes of pioneer fibers develop, yields cerci in which sensory nerves are not organized to form normal bundles within the cercus. A brief report of the principal finding has been published earlier (Edwarảs et al., 1979).

\section{Materials and Methods}

Eggs of Acheta domesticus were obtained and cultured essentially as described by Edwards and Chen (1979). 
Embryos selected for electron microscopy were fixed for a minimum of $2 \mathrm{hr}$ in a mixture of $2 \%$ paraformaldehyde and $0.5 \%$ glutaraldehyde in Millonig's phosphate buffer, followed by postfixation in $2 \%$ osmium tetroxide in Millonig's buffer. Tissues were embedded in Epon 812 . Sections for light microscopy were stained with Richardson's stain (Richardson et al., 1960). Thin sections were stained with saturated uranyl acetate and lead citrate (Reynolds, 1963) and examined with a Philips 300 electron microscope.

For microlaser lesions, selected eggs at appropriate stages were transferred to a drop of saline on a microslide and covered with a quartz coverslip. The laser microbeam system employed a neodymium Yag laser as described elsewhere (Berns et al., 1979). The 265-nm beam of a frequency-quadrupled Yag laser was reflected into a Zeiss photomicroscope equipped with quartz optics. The output of the laser at $265 \mathrm{~nm}$ was $400 \mathrm{~W}$ and the phase duration of each laser shot was 80 nsec. Various neutral density filters were used to attenuate the beam so that the beam intensity was sufficient to kill the target cells without destroying neighboring tissue. The energy density of the 1- to $2-\mu \mathrm{m}$ focused laser spot was between 10 and $50 \mu \mathrm{J}$. A quartz $\times 32$ Ultrafluar glycerin immersion objective was positioned with the cercal rudiment in focus and aligned for irradiation while under observation on a television monitor. A cross-hair on the monitor screen denoted the point of laser focus in the cercus. During irradiation, the embryo was moved and the focus was altered to ensure that the beam traversed appropriate epithelial cells. Where damage of the chorion and serosa were avoided, little damage was observed outside the target tissue, but damage to egg membranes, which occurred frequently when the cercal rudiment was in contact with them at the time of irradiation, caused rapid blackening and subsequent adhesion of embryonic tissue to the serosa. The resulting embryos were deformed and were not used for studies of nerve distribution. After lesioning, eggs were placed singly in the wells of an LKB electron microscope grid holder in a drop of saline and maintained in a moist chamber at $28^{\circ} \mathrm{C}$. They were examined periodically under a dissecting microscope, and the saline was changed every 1 or 2 days. Transfer of eggs to the surface of moist filter paper late in development allowed for normal eclosion.

\section{Results}

Laser lesions were made on cercal rudiments of embryos at stages 19 through 22 (Edwards and Chen, 1979) with the objective of ablating pioneer fiber cell bodies or their precursors before, or shortly after, the formation of their centripetal processes. Since the exact time of axon formation cannot be determined precisely in the intact embryo, a range of embryos was irradiated, some of which preceded the time at which first pioneer fibers are detectable at stage 20 . Several embryos were irradiated during the rapid phase of cercal elongation (stages 21 to 23). The general effects of laser irradiation on the cercal rudiments are described below as a context for the analysis of effects on the developing sensory system.

\section{Effects of focal laser irradiation on cercal tissue}

The distance between the cercal rudiment and enclosing egg membranes changes during development and varies among individual embryos. In embryos where the cercal tissue lay close to the egg membranes at the time of irradiation, adhesions developed at the lesion site and the subsequent development of the abdomen was arrested. Blackening, presumably due to polyphenol synthesis, developed at the wound site and became distributed throughout the embryonic fluid only if the serosal membrane was damaged by irradiation.

Localized lesions in the region of the cercal tip reduced the length of the cercus (Fig. 1a), while more basal lesions eliminated or severely reduced cercal development (Fig. 1c). Where massive lesions were sustained, the cercus failed to develop; regeneration of damaged cerci did not occur during embryogensis. Occasional duplication of the normally single sub-basal clavate mechanoreceptor hair (Fig. 1c) was the only morphogenetic effect observed in the integumental structure.

The effects of apical lesions designed to eliminate presumptive pioneer fiber cell bodies included retardation of elongation on that side so that the normal cercus tended to grow across the midline, curving over its lesioned partner. This did not affect cuticular development (Fig. 1d) or the differentiation of sensilla, although the numbers of sensilla were reduced in proportion to the loss of tissue. The cerci selected for ultrastructural studies, in most cases, were modified only in general form at the apex, with minimal modification of the basal shaft of the cercus where thin sections were taken for nerve mapping.

Visible effects on the tissue at the time of irradiation, notably a localized increased opacity in the zone of the lesion, made it possible to monitor the lateral extent of the lesion (Fig. 1e). Since the depth of the zone of cell disruption was about $5 \mu \mathrm{m}$, a single traverse could be made which affected cells on one side of the cercus only.

At the ultrastructural level, the immediate effect of the lesion is manifested in a disruption of most intracellular membranes, although the nuclear envelope frequently survives (Fig. $2 a$ ). Within $24 \mathrm{hr}$, collapse of the affected cells is accompanied by condensation of chromatins within the nuclei, swelling of the remaining mitochondria, and clumping of the remaining cytoplasmic contents (Fig. 2b). Hemocyte-like cells appear in the region of the wound within $24 \mathrm{hr}$ and become filled with lysosomes. Such cells characteristically have elaborately folded profiles, with multiple invaginations (Fig. $2 c$ ). Cellular debris is largely removed from the wound site within 2 to 3 days, and intact neighboring cells join to restore a continuous epithelium. Lysosomes persist in hemocytes throughout development and postembryonic stages and in trichogen cells (Fig. $3 a$ ). The embryonic hemocytes appear to be mobile, for lysosome-laden cells appeared in the untreated cercus within $24 \mathrm{hr}$ of irradiation. Where laser lesions damaged established pioneer fiber bundles, the distal segments degenerated rapidly (Fig. $3 b$ ), but the surrounding glial cells showed no marked short term reaction. The destruction of pioneer fibers in the cercus was followed within $24 \mathrm{hr}$ by the 

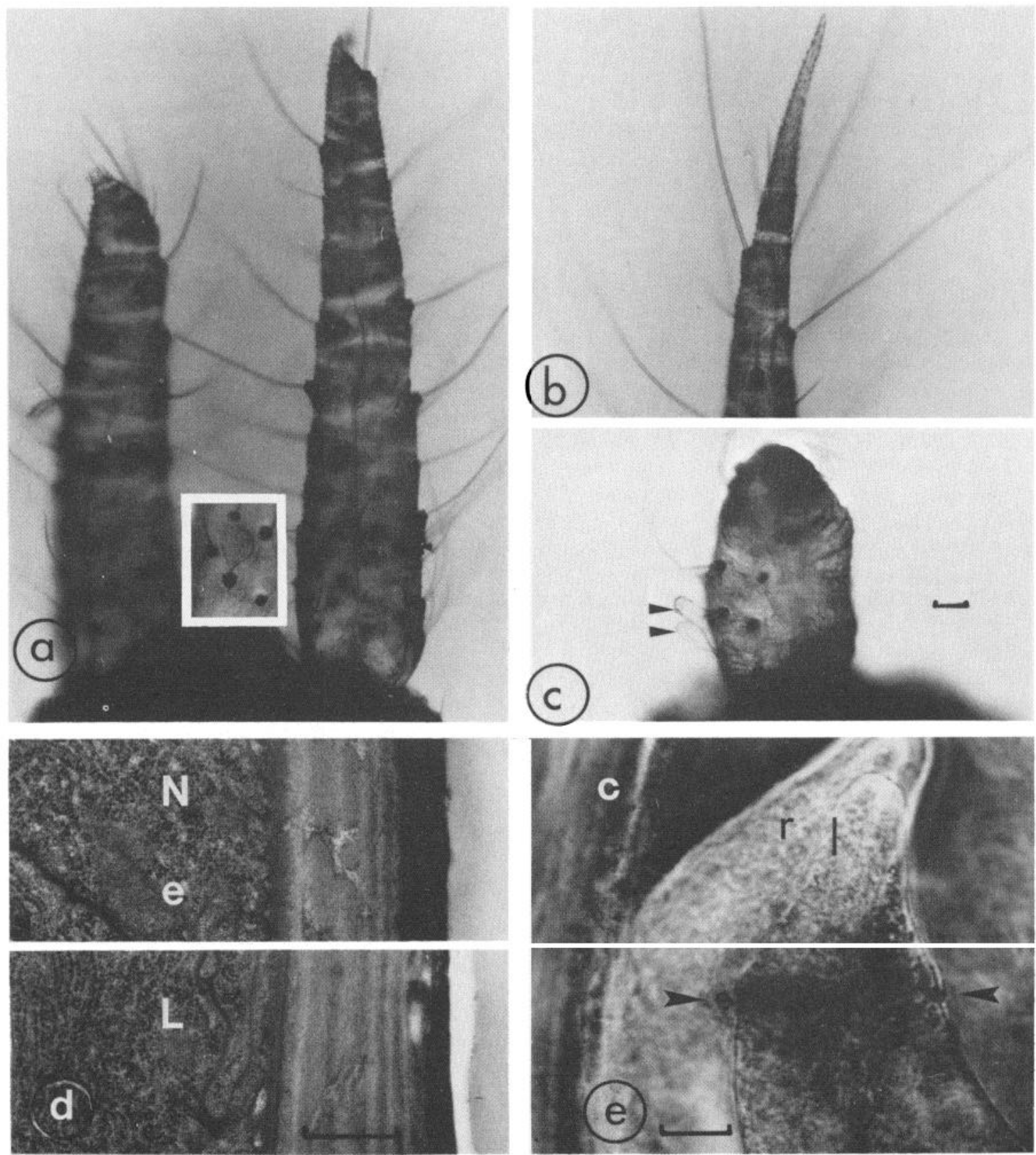

Figure 1. $a$, Cerci of newly hatched first instar Acheta larva. The left cercal rudiment was irradiated before differentiation of pioneer fibers (stage 20). Apical development was suppressed in both cerci as compared with normal cerci (b). Inset, single clavate hair situated on medial surface at base of first instar cercus. Scale for $a, b$, and $c: 50 \mu \mathrm{m}$. $b$, Apical segment of normal first instar cercus. $c$, Strongly suppressed cercus lesioned at stage 20. Development of the entire distal region is suppressed. The normally single clavate hair near the medial base is duplicated (arrows). $d$, Comparison of cuticular structure from integument of normal $(N)$ and lasered $(L)$ areas from an embryo near hatching. The number and thickness of the cuticular laminae are closely comparable. $e$, epidermal cell. Scale: $0.5 \mu \mathrm{m}$. $e$, Right $(r)$ and left $(l)$ cercal rudiments immediately after irradiation. The lesion site on the left cercus lies between the arrows. Chorion $(c)$ is at left margin. Cercal tips are at upper right margin. Scale: $10 \mu \mathrm{m}$.

Figure 2. a, Subapical transverse section of embryonic cercus (about stage 20) fixed 5 min after irradiation. Lumen (lu) is at the left. The path of the focal point passed through cells on the upper right, disrupting most intracellular structures but leaving nuclear membranes intact. The investing lamina $(l a)$, the so-called first embryonic cuticle, remains intact. Scale for $a$ and $b$ : 1 $\mu \mathrm{m}$. $b$, Detail of cercal epithelium $24 \mathrm{hr}$ after irradiation. Below the investing lamina $(l a)$, chromatin of nuclei is clumped ( $c$ ). Surviving mitochondria $(\mathrm{m})$ are swollen. The surface of intact cells has become highly folded. $c$, Detail of hemocytic-like cell packed with lysosomes $(l y)$. This cell type, which may also be the source of glia that surround pioneer fibers, assemble in the region of irradiation within $24 \mathrm{hr}$ and subsequently are found in the state shown here in other regions of the embryo. Scale: 0.5 $\mu \mathrm{m}$. 

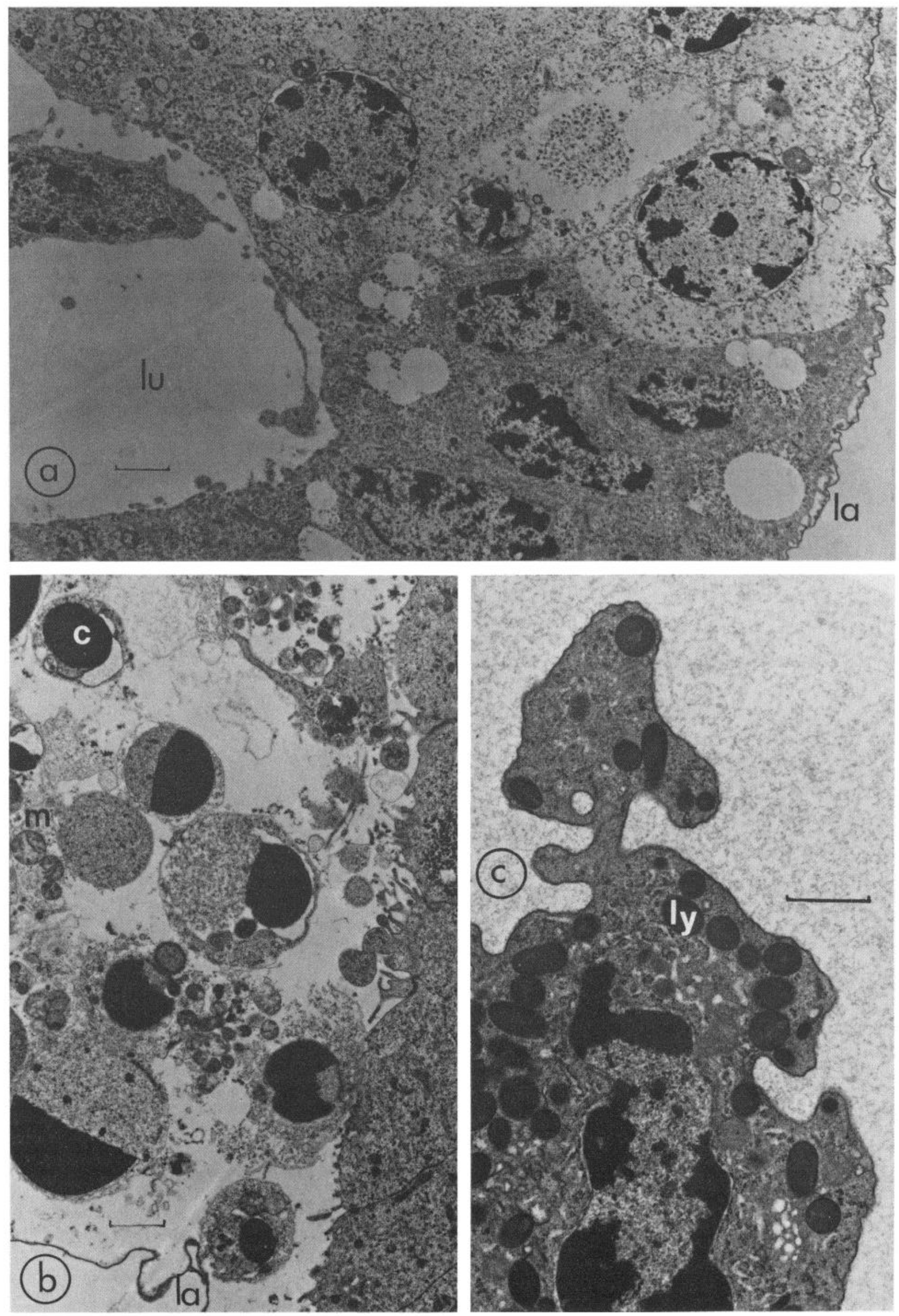

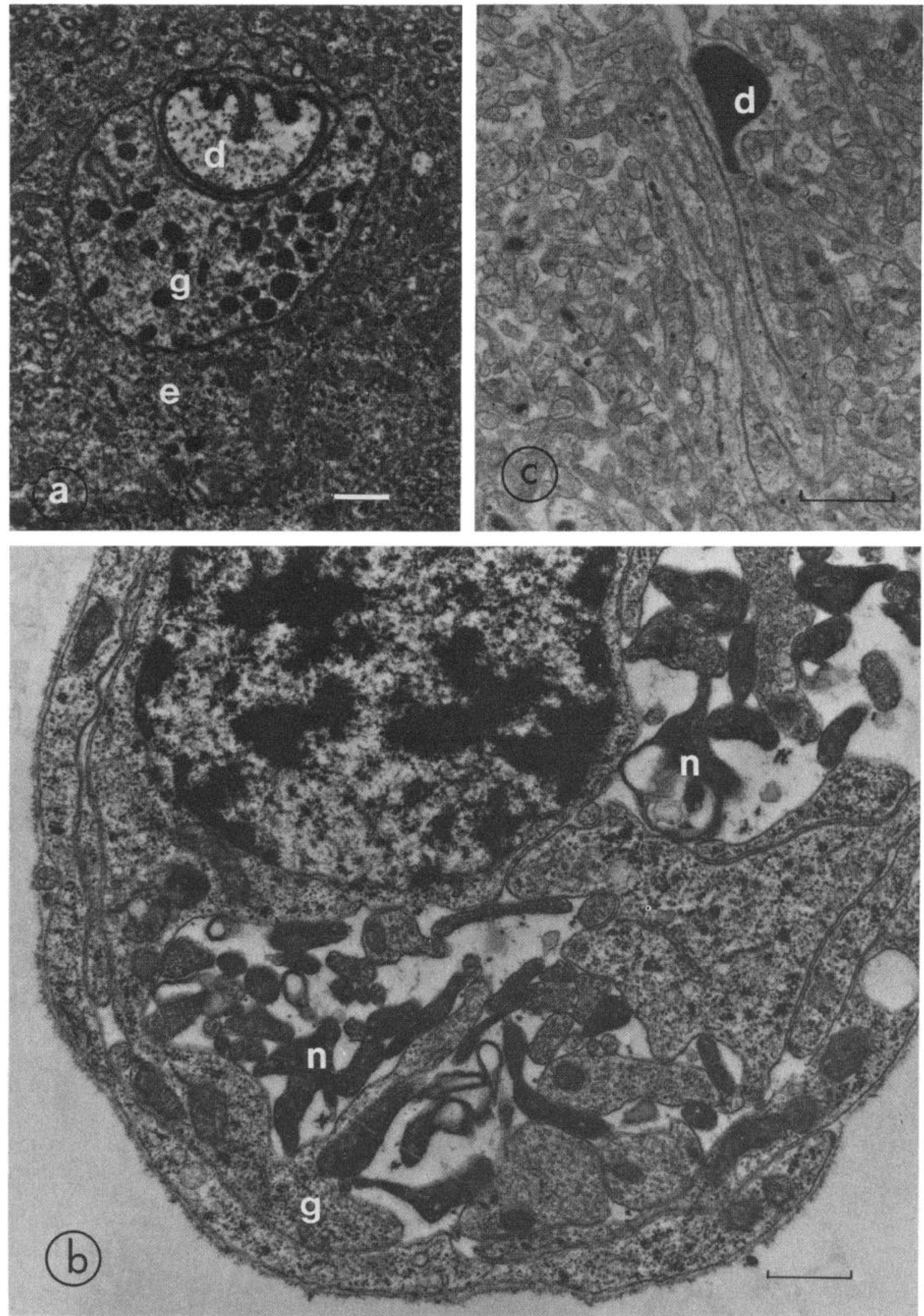

Figure 3. $a$, Dendrite $(d)$ of mechanoreceptor (filiform hair) in irradiated cercus surrounded by trichogen cell $(g)$ containing lysosomes comparable to those in Figure 2c. The surrounding epidermal cell $(e)$ contains no comparable lysosomes. Scale for $a$ and $c: 1 \mu \mathrm{m}$. $b$, Degenerating pioneer fibers in cercal tract $12 \mathrm{hr}$ after lesion. While the hitherto electron-lucent cells become densely osmiophilic $(n)$, the surrounding glia $(g)$ show no significant change. Scale: $0.5 \mu \mathrm{m}$. $c$, Subterminal degeneration $(d)$ of pioneer fiber within neuropile of embryonic terminal abdominal ganglion. 
appearance of dense osmiophilic figures in the ventral neuropile of the terminal ganglion (Fig. $3 c$ ). The similarity to degeneration figures described for crickets (Edwards and Palka, 1974) and elsewhere (e.g., Stocker et al., 1976) suggests that these are the terminal or subterminal segments of pioneer fibers.

\section{Effects of irradiation on the organization of pioneer fibers and cercal sensory nerves}

The assessment of effects of apical lesions on nerve growth was made on embryos that were allowed to approach complete embryonic development when they were fixed for electron microscopy. The distribution of sensory nerve axons in the irradiated cercus was compared with the normal member of the pair. The conformation of the sensory nerves within the cercus has been described elsewhere (Edwards and Chen, 1979). In essence, a mid-dorsal and midventral nerve in each cercus lie in contact with the basal lamina of the cercal epithelium. Axons from sensilla distributed over the cercus reach the dorsal and ventral nerves by the shortest path where they join more distal axons. Shortly before embryonic development is completed, the dorsal and ventral nerves detach from the basal lamina and come to lie in the hemocoel. They remain as separate nerves within the cercal lumen but coalesce as they leave the cercus to form the single cercal sensory nerve. The following description of treated cerci is based on a comparison of their nerve distribution.

Effects of earliest lesions. Of nine embryos irradiated before termination of katatrepsis movements and before pioneer fiber formation, one was fixed for electron microscopy 1 day after treatment. No differentiation of pioneer fiber tracts was evident nor had glial cells aggregated in mid-dorsal and midventral position of the presumptive pioneer tracts.

Of the eight fixed later in embryogenesis, four had multiple axon bundles in the treated cercus (Fig. 4, $a$ and $b$ ), while the untreated member of the pair showed the normal mid-dorsal and midventral configuration (Fig. 4c) that is identical to that of a normal cercus (Fig. $4 d$ ). In the absence of dorsal and ventral pioneer tracts, subsequently differentiating sensory axons appear to have wandered within the cercus and aggregated with other sensory axons encountered during their growth. In two cases where the early irradiation was restricted to the dorsal side of the cercal rudiment, no cercal sensory nerve developed on that surface, and the axon number was increased in the remaining nerve within that cercus (Fig. $5 b$ ). Most of the multiple bundles had glial envelopes (Figs. $4, a$ and $b$ and $5 a$ ), but several smaller groups did not.

Lesions at stage $21+$. Eight embryos lesioned at the time of, or shortly after, pioneer fiber differentiation were fixed for electron microscopy. Of these, three were fixed immediately after lesioning in order to assess the immediate effects and extent of the irradiation and are described above. One embryo, fixed 3 days after irradiation, showed no evidence of pioneer fiber formation. The remaining four embryos, fixed at or near completion of embryogenesis, all showed the normal configuration of dorsal and ventral bundles within the cercus (Fig. 5, $c$ and $d$ ).
Lesions during cercus elongation (stages 22 and 23)

Four embryos were fixed at hatching after receiving cercal lesions during the latter half of cercal elongation. Of these, three had normal cercal nerves. The fourth, which received a severe lesion resulting in distortion of the cercus, had multiple nerve bundles within the cercus comparable to those seen in embryos irradiated at stages 19 to 21 .

Three embryos were fixed 1 day after lesioning. In all cases, the axons were degenerating within the cercal nerve bundle on the lesioned side, but the glial sheath showed no significant change in ultrastructural appearance (Fig. $3 b$ ).

In a comparison of all cases described above, three general categories can be recognized. The nerve distribution was either normal, asymmetric, or multiple in embryos lesioned before the termination of katatrepsis movements. Multiple bundles were found in only one other embryo which was irradiated late in elongation and in which the cercus was distorted during development. Asymmetric patterns of nerve fiber distribution appear to have been due to the elimination of pioneer fiber on one side of the cercus only. A lesion track that did not pass through the entire target area on both sides of the apex of the cercus would produce this result.

Later lesions generally did not affect the conformation

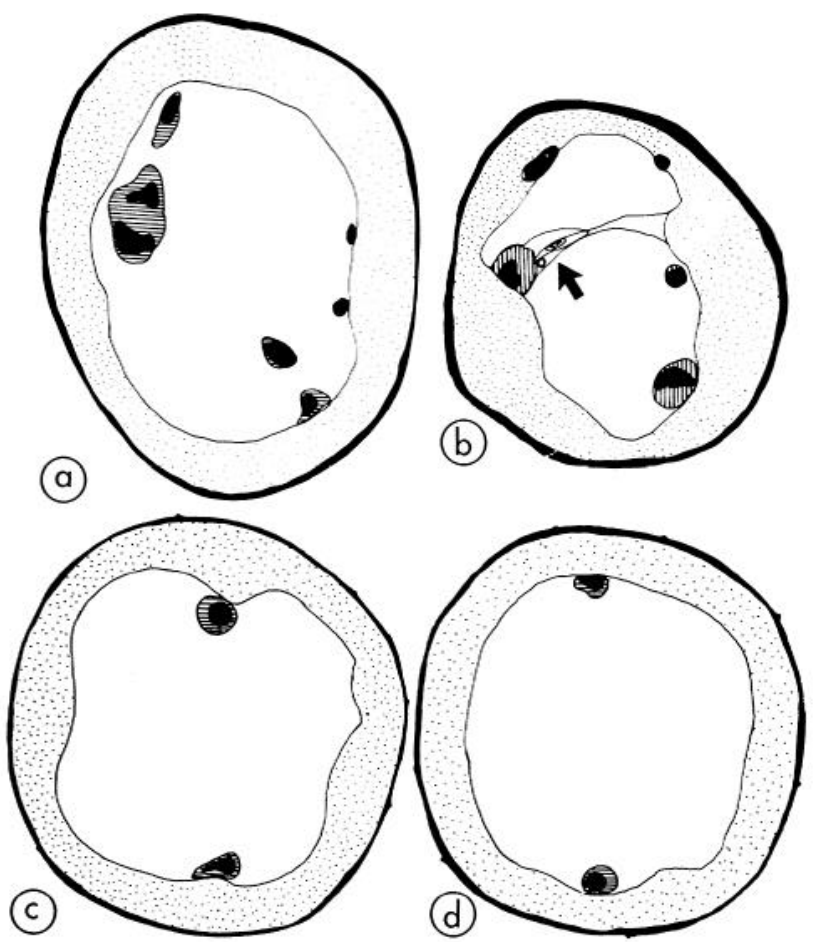

Figure 4. Tracings of cerci based on electron micrograph montages. $a$ and $b$, Multiple sensory nerves in cerci that were irradiated before formation of pioneer tracts. $c$ shows the position of the chordotonal organ shown in Figure $5 a$. The area of axon bundles is shown in black. Epidermal cells are stippled and the surrounding cuticle is shown black. $c$, Normal configuration of cercal nerves in cercus irradiated after the formation of the pioneer fiber pathways. $d$, Normal distribution of cercal sensory nerves at mid-dorsal and midventral positions shortly before they detach. 

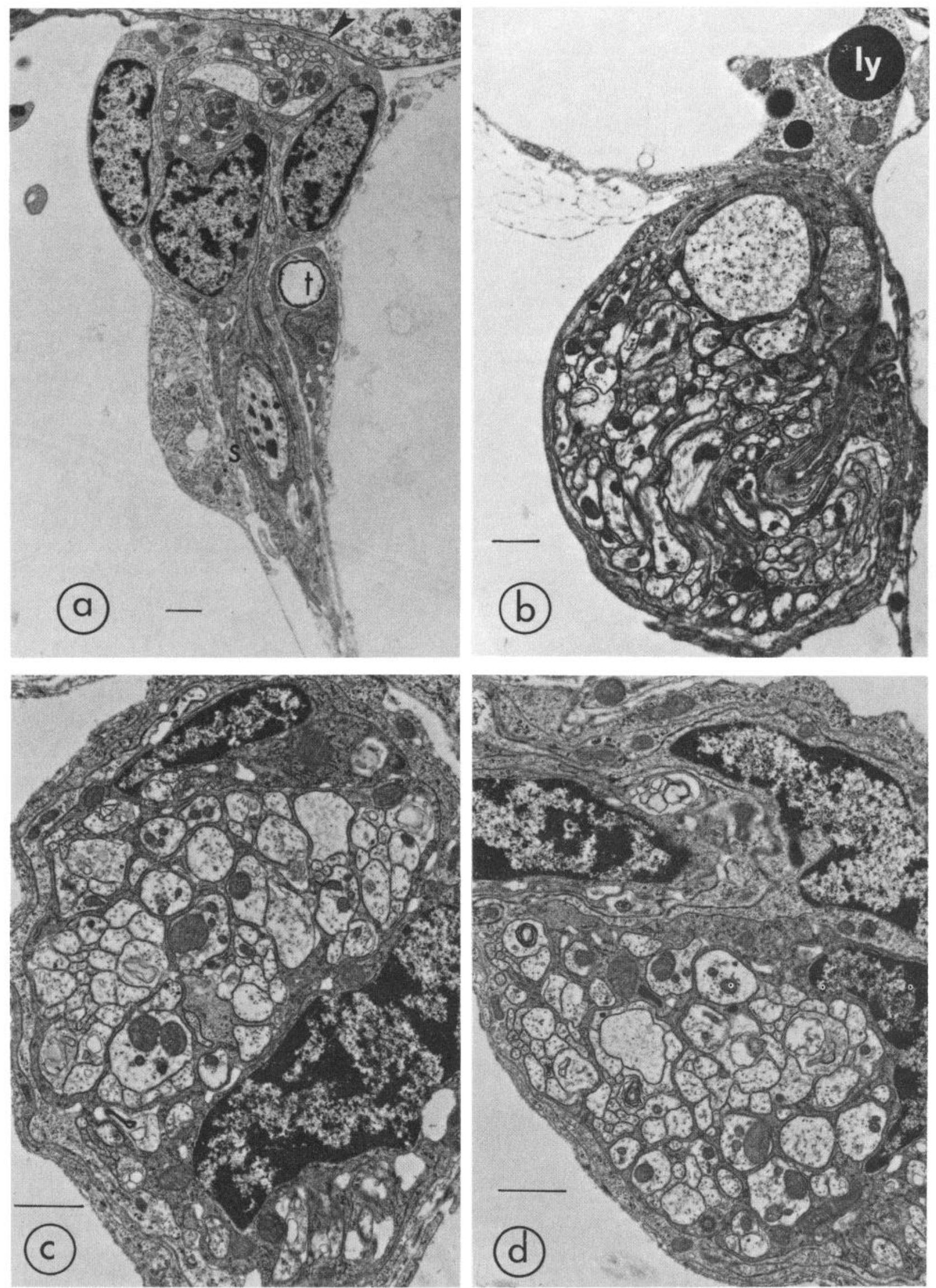
TABLE I

Summary of cercal sensory nerve configuration within embryonic cerci subjected to laser lesions at successive stages of development

\begin{tabular}{cccc}
\hline Stages & Nerves Normal & Multiple Nerves & Nerves Absent \\
\hline $19-21$ & 2 & 4 & 2 \\
$21-22$ & 4 & 0 & 0 \\
$22-23$ & 3 & 1 & 0
\end{tabular}

of the cercal nerves within the cercus. The results are summarized in Table I.

\section{Discussion}

The paths taken by growing axons during embryogenesis in insects are laid down early. In the first axons in appendages of grasshoppers (Bate, 1976; Keshishian, 1980) and crickets (Edwards, 1977; Edwards and Chen, 1979), the pioneer fibers occupy constant positions and establish pathways along which subsequently differentiating sensory axons travel to reach their central destinations. Similar functions are served by temporary processes of central neurons (Goodman et al., 1979). Our objective in this study was to determine whether this proposed guidance role is essential to the organization of the functional peripheral nerves during their development in the last $15 \%$ of embryogenesis. The use of microlaser lesions provided the potential for localizing damage to the site of pioneer fiber cell bodies, thus reducing the possibility that more extensive tissue damage alone might cause disruption of sensory axon development. Ultrastructural examination of cercal rudiments immediately after lesion indicated that the lesions were indeed local, except for the appearance of lysosomes in hemocyte-like cells which subsequently were found to be distributed through the affected and the neighboring normal cercus. Later lesions did not affect the normal development of cercal sensory nerves except for a reduction in the number of sensilla related to reduced total area of the smaller cercus, and we conclude that a localized lesion per se had no effect in sensory differentiation. Epidermal function appeared normal as judged by comparison of the sequences of cuticular layers formed in lesioned and normal cerci and neighboring abdominal integument.

The largest proportion of modified cercal sensory nerves were found in cerci where lesions were produced at or before the formation of the pioneer fibers. The presence of multiple bundles of sensory axons that showed no particular orientation within the cercus and the absence of bundles in the normal position indicate that normal organization of the cercal sensory nerves does not proceed if the pioneer fiber tract is not laid down.
The pioneer fibers become enveloped by companion cells that occupy the position of glia in the final nerve and which may be considered pioneer glia without necessarily implying that they take up their positions before the axons grow. The precise temporal relationship between the differentiation of the pioneer fibers and their glia is not known yet and it may be that the glial cells are in the vicinity of the dorsal and ventral midlines of the cercal lumen before the formation of the pioneer fibers. If so, they seem to be unable to function alone as organizers of the sensory bundles. The outcomes of later lesions to the pioneer fiber tracts, in which the axons degenerate but glia persist, show that the glial conduit once formed during the early association of pioneer fiber processes and glia can function as an organizing focus for the functional sensory fibers.

We have noted earlier (Edwards and Chen, 1979) that the mid-dorsal and midventral lines of the basal lamina underlying the cercal epithelium invariably serve as the surface on which the pioneer fibers make their transit to the base of the cercus. The pioneer fibers remain in contact with this surface until late in embryogenesis when the functional nerves of sensory axons have traversed the length of the cercus en route to the center. The glia retain their association with this surface after distal lesions kill the pioneer fibers and they can persist to form the aggregation site for the functional nerves.

Some uncertainty persists concerning the capacity of the pioneer cells to regenerate processes in cases where the axon was severed by the lesion but the cell body persisted. This possibility cannot be eliminated yet, but the more extreme case of regulation following loss of the cell bodies of pioneer fibers appears most unlikely for two reasons: first, because pioneer tracts did not appear in expected positions where the terminal lesion had clearly damaged the site of pioneer fibers and second, because the capacity for regeneration or regulation of the embryonic cercus appears to be restricted. Wound healing occurs in early massive lesions, but conpensatory growth does not occur and a normal cercus is never formed even though 50 to $60 \%$ of developmental period is available.

The persistence of the pathway between the base of the cercus and the ganglion was not investigated in this phase of the study in which attention was restricted to events within the cercus. We note, however, that degenerating axon profiles were observed in ventral positions within the embryonic ganglia after lesions of the cercal apex in stage 20 , that is shortly after differentiation of the pioneer fibers, a finding in accord with Shankland's (1979) observation on the central projection of cercal pioneer fibers in the grasshopper Schistocerca nitens. Connections between cerci and the center were estab-

Figure 5. a, One of several axon bundles (arrow top right) from lesioned cercus shown in Figure $4 b$. The scolopale ( $s$ ) of a chordotonal organ within the cercus lies adjacent to the nerve. A trachea $(t)$ lies in contact with the chordotonal organ. Scale for $a$ to $d: 1 \mu \mathrm{m}$. $b$, Cercal sensory nerve within cercus in which the pioneer fiber pathway of one side was eliminated by laser lesion. In this case, all sensory axons have aggregated with the remaining tract. Lysosomes $(l y)$ are in neighboring hemocyte. $c$, Normal cercal sensory nerve from normal cercus fixed shortly before hatching. $d$, Normal cercal nerve formed in cercus after irradiation at stage 21 after the differentiation of the pioneer fiber tract. 
lished in animals lesioned after formation of the pioneer fibers but before differentiation of sensilla, suggesting, but not proving, that the pathway to the ganglion is preserved under these conditions.

\section{References}

Bate, C. M. (1976) Pioneer neurons in an insect embryo. Nature 260: 54-56.

Berns, M. W., L. K. Chong, M. Hammer-Wilson, K. Miller, and A. Siemens (1979) Genetic surgery by laser: Establishment of a clonal population of rat kangaroo cells $\left(\mathrm{PTK}_{2}\right)$ with indirected deficiency in a chromosomal nucleolar organiser. Chromosoma 73: 1-8.

Edwards, J. S. (1977) Pathfinding by arthropod sensory nerves. In Identified Neurons and Behavior of Arthropods, G. Hoyle, ed., pp. 484-493, Plenum Press, New York.

Edwards, J. S., and S.- W. Chen (1979) Embryonic development of an insect sensory system, the abdominal cerci of Acheta domesticus. Wilhelm Roux's Arch. Dev. Biol. 186: 151-178.

Edwards, J. S., and J. Palka (1974) The cerci in abdominal giant fibers of the house cricket Acheta domestica. I. Anatomy and physiology of normal adults. Proc. R. Soc. Lond.
(Biol.) 185: 83-103.

Edwards, J. S., M. W. Berns, and S.- W. Chen (1979) Laser lesions of embryonic cricket cerci disrupt guidepath role of pioneer fibres. Soc. Neurosci. Abstr. 5: 158.

Goodman, C. S., M. Bate, and N. C. Spitzer (1979) Origin transformation and death of neurons from an identified precursor during grasshopper embryogenesis. Soc. Neurosci. Abstr. 5: 161.

Keshishian, H. (1980) The origin and morphogenesis of pioneer neurons in the grasshopper metathoracic leg. Dev. Biol., in press.

Reynolds, E. S. (1963) The use of lead nitrate at high $\mathrm{pH}$ as an electron-opaque stain in electron microscopy. J. Cell Biol. 17: 208-212.

Richardson, K. C., L. Jarrett, and E. H. Finke (1960) Embedding in epoxy resins for ultrathin sectioning in electron microscopy. Stain Technol. 35: 313-323.

Shankland, M. (1979) Development of pioneer and sensory afferent projections in the grasshopper embryo. Soc. Neurosci. Abstr. 5: 178.

Stocker, R. F., J. S. Edwards, J. Palka, and G. Schubiger (1976) Projection of sensory neurons from a homestic mutant appendage, antennapedia in Drosophila melanogaster. Dev. Biol. 52: 210-220. 